\title{
¿Cuál es la seguridad de un programa de ejercicio, como intervención, durante la hemodiálisis para el paciente con enfermedad renal crónica?
} What is the safety of an exercise program, as an intervention, during hemodialysis for the patient with chronic kidney disease?

\author{
๑ Oscar Mauricio Sarmiento Becerra ${ }^{1}, \odot$ Angélica María Puentes Salazar ${ }^{1}$, \\ (-) Andrés Eduardo Hernández ${ }^{1}$ \\ 'Hospital Infantil Universitario de San José, Bogotá, Colombia
}

\begin{abstract}
Resumen
La enfermedad renal crónica es una patología de alto costo, disminuye la calidad de vida, especialmente, la de los pacientes en hemodiálisis, es importante implementar estrategias para reducir su impacto clínico y económico. El ejercicio intradiálisis ha mostrado la reducción en la mortalidad por cualquier causa y mejoría en la calidad de vida. Por medio de esta revisión de la literatura disponible en las bases de datos Pubmed, OVID, VHL, Clinical Key y LILACS, se busca determinar la seguridad del ejercicio intradiálisis, se han revisado las características de la población intervenida, los tipos de intervención y los eventos adversos. Se incluyeron 8 artículos de la búsqueda sistemática y 15 más por búsqueda manual. Se encontró que la población era en su mayoría adulta (con edades entre los 19 a 88 años) y solo un estudio reportó una población más joven (edades entre los 9,1 a 24,2 años); la proporción entre géneros fue similar. Se encontró que la intervención más frecuente fue el ejercicio cardiovascular realizado con sistemas de pedales, de intensidades bajas a moderadas, con una frecuencia de 2 a 3 sesiones semanales durante mínimo 2 meses. Algunos estudios no reportaron efectos adversos, pero los que sí, mencionan complicaciones en el sistema cardiovascular (hipotensión/ hipertensión) y en otras se manifiesta lesiones músculo esqueléticas (poco frecuentes), pero ninguna considerada severa o frecuente. Con la información disponible se concluyó que el ejercicio intrahemodiálisis parece ser una intervención segura.

Palabras clave: terapia por ejercicio, diálisis renal, seguridad.
\end{abstract}

doi: http://dx.doi.org/10.22265/acnef.6.1.328

\begin{abstract}
Chronic kidney disease is a high-cost pathology; it has a negative impact on the quality of life, especially in those who underwent hemodialysis. Consequently, it is important to develop strategies to decrease the clinical and economic impact. Intradialytic exercise is a complementary intervention that has shown a decrease in mortality and improvement in the quality of life in these patients. This paper aimed to review the available literature and determine the security of intradialytic exercise, look for population characteristics, training characteristics and the occurrence of adverse effects and their severity. This document is a topic review with a systematic search on Pubmed, OVID, VHL, Clinical Key and LILACS. 8 articles appeared after a keyword search with 15 additional papers discovered by manual research by the authors, the population included adults between 19 and 88 years of age with only 1 paper including a younger population from 9 to 24 years of age. Sex distribution was similar between male and female. Endurance training with a pedal system was the most frequent intervention. The frequency of intervention was 2 to 3 times per week at least for 2 months, and the intensity was low to mild. Some trials mentioned no adverse effect. Those with adverse effects reported cardiovascular alterations (hypotensive/hypertensive episodes), muscular injuries, but none of the adverse effects were considered severe or frequent. With the available information, the authors conclude that intradialytic exercise will be a secure intervention.
\end{abstract}

Key words: exercise therapy - renal dialysis - safety.

doi: http://dx.doi.org/10.22265/acnef.6.1.328

Citación: Sarmiento Becerra OM, Puentes Salazar AM, Hernández AE ¿Cuál es la seguridad de un programa de ejercicio, como intervención, durante la hemodiálisis para el paciente con enfermedad renal crónica?- Revisión narrativa. Rev. Colomb. Nefrol. 2019;6(1):35-47. doi: http://dx.doi.org/10.22265/acnef.6.1.328 Correspondencia: Oscar Mauricio Sarmiento Becerra, omsarmiento@fucsalud.edu.co

Recibido: $26.11 .18 \cdot$ Aceptado: 12.12 .18 


\section{Introducción}

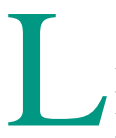

a enfermedad renal crónica (ERC) es una patología no transmisible que tiene un mal pronóstico clínico y que por su cronicidad deriva en una alta tasa de discapacidad con un gran impacto económico. En Colombia, por más de 20 años, la Cuenta de Alto Costo (CAC) la clasifica como patología de "alto costo", sin embargo, en esos reportes solo incluían a la terapia dialítica, como intervención para el manejo de esta población; recientemente están incluyendo una serie de recomendaciones e intervenciones encaminadas a mejorar la calidad de vida relacionada con la salud, con énfasis en el mantenimiento de un peso adecuado y en el aumento de la capacidad funcional a través del ejercicio, tal y como lo indican las directrices del Kidney Disease Outcomes Quality Initiative (K-DOQI) ${ }^{1-3}$.

En un estudio descriptivo de pacientes en hemodiálisis (HD), con población colombiana, se encontró que el 97,5 \% tenía una vida sedentaria, resultados similares a lo reportado por Paneye y colaboradores en su estudio, donde encontró que el $94 \%$ de su población objeto tenía niveles bajos de actividad física, el 73,2 \% tenía un índice de masa corporal (IMC) dentro de parámetros normales, pero cuando se observó la composición corporal, presentaron un porcentaje de masa muscular bajo y de masa grasa alto, datos que permiten inferir la importa ncia de una intervención con ejercicio en esta población ${ }^{4}$.

La prevalencia de ERC ha aumentado progresivamente a nivel mundial; para nuestro país en el último informe de la CAC (2017) se reportó que en el último año, 38.869 personas requirieron de alguna terapia de reemplazo renal (TRR), lo que corresponde a una prevalencia de 78,9 por cada 100.000 habitantes, con una población económicamente productiva y relativamente joven y un promedio de edad de 55,7 años $^{1,2}$. Lo anterior amerita plantear estrategias para disminuir el impacto clínico y económico de esta patología renal crónica.

En la literatura se encuentran estudios con diferentes intervenciones y entre esas, en algunos, se incluye el ejercicio físico. Es importante destacar que con estas intervenciones se habla de la reduc- ción de la mortalidad, disminución de tasa de hospitalización, asociadas con la mejoría en el rendimiento físico (tanto en la resistencia cardiovascular como en la fuerza muscular) y cómo incide en la calidad de vida relacionada con la salud en esta población con $\mathrm{ERC}^{3,5}$. A su vez, exponen numerosas barreras para la implementación de dichos programas, como la falta de promoción del ejercicio, la financiación, la falta de tiempo para realizarlo, y las dudas asociadas a la seguridad de esta intervención (posibles riesgos relacionados con la práctica de ejercicio), así como también la falta de experticia en la prescripción del ejercicio en este tipo de pacientes con riesgo aumentado por su patología de base y de las comorbilidades asociadas ${ }^{3}$.

En una población que realiza un plan de ejercicios regularmente, el riesgo más común es la lesión músculo-esquelética; sin embargo, los más graves son los de origen cardiovascular en cualquier presentación, como por ejemplo: arritmias, enfermedad coronaria y hasta la muerte súbita asociada. Se debe tener en cuenta que dicho riesgo aumenta con varios factores como la edad, el estado de salud previo, los antecedentes de riesgo cardiovascular, e incluso la intensidad a la cual se realiza el ejercicio (a mayor intensidad el riesgo es mayor y si el ejercicio es considerado máximo es mucho más riesgoso que si es de intensidad submáxima) entre muchos otros ${ }^{6}$.

Los trabajos de fuerza a intensidades adecuadas y bien controladas, aumentan la fuerza y funcionalidad muscular, reduciendo el riesgo de caídas y promueven la mineralización ósea, lo que representa un beneficio para los pacientes con ERC. Es conocido, que la población de enfermos renales crónicos tiene una alta prevalencia de factores de riesgo para la enfermedad cardiovascular, la cual en muchos casos ya está establecida, sin embargo, el riesgo con una intervención que involucre el ejercicio en su manejo, probablemente no sea significativamente mayor al riesgo de otras poblaciones sometidas a pruebas diagnósticas de enfermedad cardiovascular (como por ejemplo la prueba de esfuerzo $)^{6}$, o de usuarios de servicios de rehabilitación cardiaca, donde son ampliamente manejados, incluso con cargas de trabajo físico intenso. 
Esta revisión pretende determinar el perfil de seguridad de los programas de ejercicio en los pacientes en hemodiálisis, los eventos adversos asociados y la severidad cuando se manifiestan.

\section{Métodos}

Este documento es una revisión de tema. Las bases donde se realizó la búsqueda fueron: Pubmed, OVID, VHL, Clinical key y LILACS. Los términos de la estrategia en español fueron: ejercicio terapéutico, diálisis renal, seguridad y en inglés: exercise therapy, renal dialysis, safety. Se incluyeron ensayos clínicos, estudios piloto, revisiones narrativas y sistemáticas / meta-análisis, en donde se sometió a una intervención con ejercicio de resistencia cardiovascular y/o de fuerza a pacientes con enfermedad renal crónica, durante la hemodiálisis, y que además evaluaran como desenlace la seguridad de la intervención de estos ejercicios y también los efectos adversos (si los hubo) de la intervención intradiálisis. La búsqueda se restringió a los idiomas inglés, español y portugués. Se excluyeron artículos donde se combinaran ejercicio con medicamentos para bajar de peso o para mejorar la capacidad funcional, así como encues- tas de percepción de la actividad física donde no se realizó ninguna intervención.

Se extrajeron además datos de desenlaces como los tipos de intervención y las características de la población. Los artículos fueron seleccionados inicialmente por el título, posteriormente con lectura de resumen y texto completo. También se incluyeron por búsqueda manual artículos que aportan información relevante sobre los desenlaces que son objeto de esta revisión. Finalmente se incluyeron 23 artículos (figura 1).

\section{Resultados}

\section{Descripción de los artículos incluidos en la revisión}

De los 23 artículos incluidos, 9 son estudios clínicos aleatorizados, 4 son ensayos clínicos no aleatorizados, 3 son estudios clínicos previos y posteriores a la intervención, 3 son revisiones narrativas, 1 es un estudio piloto factorial $2 \times 2 ; 2$ son revisiones sistemáticas y meta-análisis y 1 es un estudio prospectivo intervencionista. En la tabla 1 se describe el diseño y algunas características importantes de cada artículo.

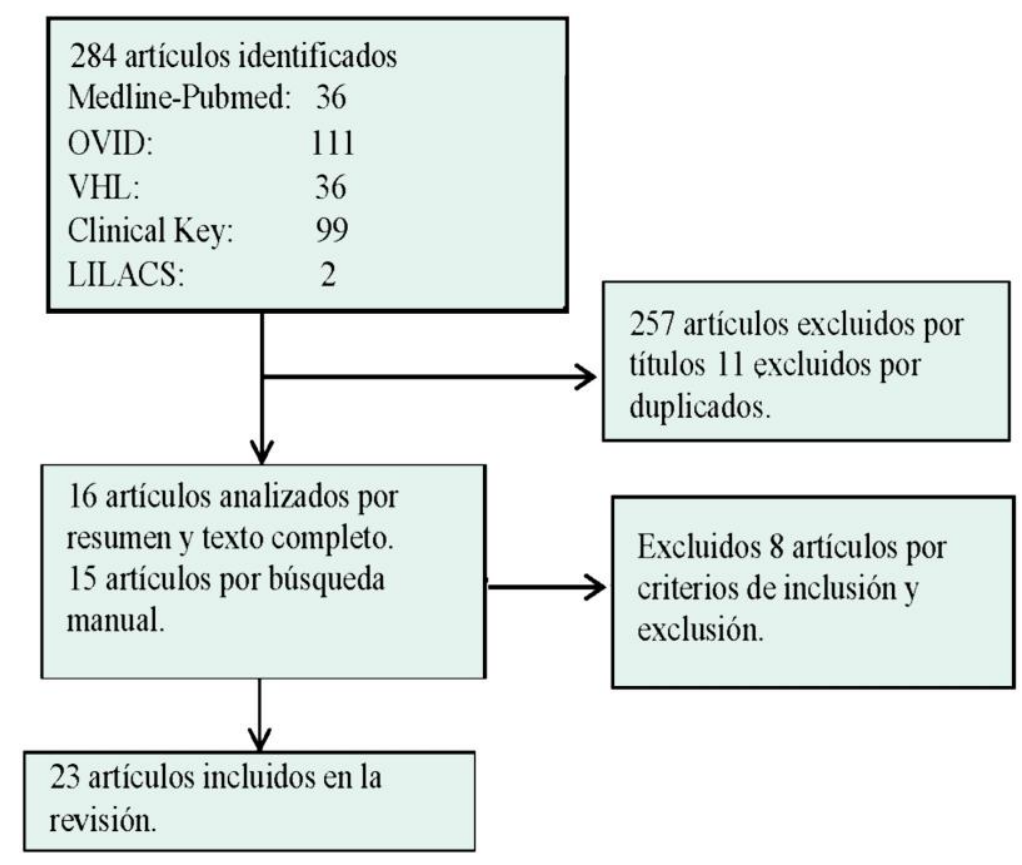

Figura 1. Flujograma de la búsqueda de artículos para la revisión. 
Tabla 1. Descripción de los artículos incluidos en la revisión.

\begin{tabular}{|c|c|c|c|}
\hline Autor. Año (Referencia) & Diseño del estudio & Idioma & $\begin{array}{l}\text { Extraído de base de } \\
\text { datos o manual }\end{array}$ \\
\hline Thompson, et al ${ }^{\top}$ & Estudio aleatorizado piloto factorial $2 \mathrm{X} 2$ & Inglés & PUBMED \\
\hline Sheng, et $\mathrm{al}^{8}$ & $\begin{array}{l}\text { Revisión sistemática y meta-análisis } \\
\text { Incluyo } 24 \text { estudios }\end{array}$ & Inglés & PUBMED \\
\hline Paglialonga, et al ${ }^{9}$ & Ensayo clínico no aleatorizado & Inglés & PUBMED \\
\hline Oh Park, et al ${ }^{11}$ & Ensayo clínico no aleatorizado & Inglés & PUBMED \\
\hline Spinola Najas, et $\mathrm{al}^{12}$ & $\begin{array}{l}\text { Revisión Narrativa. } \\
\text {-Incluyeron } 6 \text { estudios intradiálisis y } 6 \text { extradiálisis }\end{array}$ & Portugués & VHL (SciELO) \\
\hline Bohm, et al ${ }^{13}$ & $\begin{array}{l}\text { Revisión Literaria } \\
\text {-Incluyeron } 14 \text { estudios experimentales }\end{array}$ & Portugués & Manual \\
\hline Segura-Orti ${ }^{14}$ & $\begin{array}{l}\text { Revisión sistemática y Meta-análisis } \\
\text {-Incluyeron } 14 \text { estudios }\end{array}$ & Español & Manual \\
\hline V. Esteve Simo, et al., $2015^{15}$ & Estudio prospectivo unicéntrico no aleatorizado & Español & Manual \\
\hline Olvera Soto, et al., $2015^{16}$ & Ensayo clínico aleatorizado & Inglés & Clinical Key \\
\hline Parsons y King Val Vlack ${ }^{17}$ & $\begin{array}{l}\text { Revisión Narrativa. } \\
\text {-Incluyeron } 18 \text { publicaciones intradiálisis y } 16 \text { extradiálisis }\end{array}$ & Inglés & Clinical Key \\
\hline 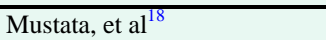 & Ensayo clínico controlado aleatorizado & Inglés & Manual \\
\hline Konstantinidou, et $\mathrm{al}^{19}$ & Ensayo clínico controlado, aleatorizado & Inglés & Manual \\
\hline Leaf, et $\mathrm{al}^{20}$ & Ensayo clínico controlado, no aleatorizado & Inglés & Manual \\
\hline Headley, et $\mathrm{al}^{21}$ & Ensayo clínico controlado, aleatorizado & Inglés & Manual \\
\hline Kouidi, et al ${ }^{22}$ & Ensayo clínico controlado, no aleatorizado & Inglés & Manual \\
\hline Kouidi, et $\mathrm{al}^{23}$ & Ensayo clínico controlado, aleatorizado & Inglés & Manual \\
\hline Storer, et al ${ }^{24}$ & Ensayo clínico, antes y después. & Inglés & Manual \\
\hline Kong, et $\mathrm{al}^{25}$ & Ensayo clínico controlado, aleatorizado & Inglés & Manual \\
\hline Painter, et $\mathrm{al}^{26}$ & Ensayo clínico controlado, aleatorizado & Inglés & Manual \\
\hline Macdonald, et $\mathrm{al}^{21}$ & Ensayo clínico, antes y después. & Inglés & Manual \\
\hline Cheema, et al., $2007^{28}$ & Ensayo clínico controlado, aleatorizado & Inglés & Manual \\
\hline DePaul, et al., $2002^{29}$ & Ensayo clínico controlado, aleatorizado & Inglés & Manual \\
\hline Koufaki, et al, ${ }^{30}$ & Ensayo clínico controlado, aleatorizado & Inglés & Manual \\
\hline
\end{tabular}

\section{Características de la población}

En algunos de los artículos revisados, la población que participa en los estudios debe cumplir unos criterios claros de inclusión, tales como ser mayor de 18 años, llevar más de tres meses consecutivos de hemodiálisis (HD), asistir tres a más veces por semana a la HD, tener buena movilidad, o no tener alteraciones que impidan la movilidad normal ${ }^{7,9,16} \mathrm{o}$ edad igual o mayor a 80 años ${ }^{15}$ y otros, son claros al enumerar los criterios de exclusión para esos estudios, como la presencia de una patología aguda que impida la participación en un programa de ejerci$\operatorname{cios}^{7}$, evento coronario agudo, imposibilidad física, o mental, contraindicaciones para realizar una prueba de esfuerzo, según la Asociación Americana de Cardiología (AHA), incluyendo también a personas con DM, con alto riesgo para desprendimiento de retina $^{11}$, o presencia de hipotensión severa considerada como tensión arterial menor de 90/70 mm Hg durante las sesiones ${ }^{15}$, también se habla de pacientes trasplantados, o con pérdida de la visión, que son también criterios de exclusión ${ }^{16}$. El estudio de Konstantinidou $^{19}$, es más selectivo e incluye como criterios de exclusión a las personas con HTA inestable o mal controlada, falla cardiaca en estadios avanzados, arritmias ventriculares Lown 3, hipercalemia persistente luego de la HD, diabetes mellitus, enfermedades hepáticas, óseas o vascular periférica. Por otra parte, la población es en su mayoría adultos, en rangos entre los 19 hasta 88 años (excepto el estudio de Paglialonga, et al. ${ }^{9}$, que incluyó a la población más joven en rangos de 9,1 a 24,2 años. La participación de mujeres fue en proporciones similares con un ligero predominio del género masculino, y en algunos se menciona, que incluyeron pacientes de raza negra y caucásica. En los estudios de Thompson, Oh Park, Segura-Orti, y Simo, et al., se menciona que dentro de las causas etiológicas de la ERC, las más frecuentes, eran la 
hipertensión arterial, la diabetes mellitus, la enfermedad poliquística renal y la enfermedad glomerular; además Olvera, et al., reportan que el $83 \%$ de la población presentó algún grado de desnutrición o mala nutrición $^{7-14}$ (tabla 2). Algunos estudios tuvieron en cuenta parámetros de laboratorio e identificaron su variación tras el programa de ejercicio (tablas 3 y 4 ).

Tabla 2. Características de las intervenciones realizadas en los ensayos clínicos revisados.

\begin{tabular}{|c|c|c|c|}
\hline Estudio & Intervención & Frecuencia & Duración \\
\hline Thompson, et al ${ }^{7}$ & $\begin{array}{l}\text { Ejercicio aeróbico: cada sesión incluyó } 5 \text { minutos de puesta } \\
\text { a punto y de vuelta a la calma en el cicloergómetro a una } \\
\text { intensidad entre } 9 \text { y } 11 \text { en escala de Borg. } \\
\text { El protocolo fue pedaleo constante durante } 15 \text { minutos con } \\
\text { progresión de } 2,5 \text { minutos cada semana. Se ajustó la } \\
\text { resistencia para lograr la intensidad objetivo de } 12 \text { a } 14 \text { en } \\
\text { escala de Borg. }\end{array}$ & No describe & 12 semanas \\
\hline Paglialonga, et $\mathrm{al}^{9}$ & $\begin{array}{l}30 \text { minutos sesiones de ejercicio intradiálisis utilizando un } \\
\text { cicloergómetro. }\end{array}$ & $\begin{array}{l}\text { Dos a tres veces } \\
\text { a la semana }\end{array}$ & 3 meses. \\
\hline Smart y Steele ${ }^{10}$ & $\begin{array}{l}\text { Estudios en población en hemodiálisis regular, intervención } \\
\text { con ejercicio vs. no ejercicio o comparando diferentes tipos } \\
\text { de ejercicio. }\end{array}$ & No describe & No describe \\
\hline Oh Park, et al ${ }^{11}$ & $\begin{array}{l}\text { Ejercicio de cicloergómetro y fortalecimiento de los } \\
\text { extensores de la rodilla. }\end{array}$ & $\begin{array}{l}2-3 \text { veces } \\
\text { por semana }\end{array}$ & 3 meses \\
\hline Bohm, et $\mathrm{al}^{13}$ & $\begin{array}{l}\text { Ejercicio con cicloergómetro durante la diálisis ( } 14 \text { ensayos } \\
\text { clínicos controlados aleatorizados) durante la primera hora a } \\
\text { los primeros } 90 \text { minutos. Intensidad entre el } 40 \%-60 \% \\
\text { del V02 pico o entre el } 50 \% \text { y } 85 \% \text { de la frecuencia } \\
\text { cardiaca máxima. }\end{array}$ & $\begin{array}{l}2-3 \text { veces por } \\
\text { semana }\end{array}$ & Entre 6 - 40 semanas \\
\hline Segura-Orti ${ }^{14}$ & $\begin{array}{l}\text { Se revisaron } 14 \text { estudios, con intervenciones principalmente } \\
\text { de ejercicio cardiovascular y en algunos estudios } \\
\text { combinados ejercicios aeróbicos y de fuerza. La intensidad } \\
\text { del ejercicio varió entre el } 50 \text { y el } 80 \% \text { del V02 pico o de } \\
\text { la frecuencia cardiaca máxima principalmente. }\end{array}$ & $\begin{array}{l}3 \text { veces por } \\
\text { semana }\end{array}$ & $\begin{array}{l}\text { Entre } 2 \text { meses y } 4 \text { años } \\
(90 \% \text { de los estudios } \\
\text { con duración entre } 3 \text { y } \\
6 \text { meses) }\end{array}$ \\
\hline V. Esteve Simo, et al $^{15}$ & $\begin{array}{l}\text { Ejercicio fisico adaptado mediante pelotas medicinales, } \\
\text { pesas, bandas elásticas y cicloergómetro en las primeras } 2 \\
\text { horas de hemodiálisis. }\end{array}$ & No describe & 12 semanas \\
\hline Olvera Soto, et al ${ }^{16}$ & $\begin{array}{l}\text { Ejercicio de fuerza durante las sesiones de hemodiálisis con } \\
\text { pesas de tobillo y bandas. }\end{array}$ & $\begin{array}{l}2 \text { veces por } \\
\text { semana }\end{array}$ & 12 semanas \\
\hline $\begin{array}{l}\text { Parsons and King Val } \\
\text { Ylack }^{17}\end{array}$ & $\begin{array}{l}\text { Ejercicio de baja y moderada intensidad, medidos por\% de } \\
\text { V02 pico, frecuencia cardiaca máxima y Borg. Mayoría de } \\
\text { ejercicio cardiovascular en cicla, algunos programas } \\
\text { combinados con fuerza. }\end{array}$ & $\begin{array}{l}2-3 \text { veces por } \\
\text { semana }\end{array}$ & 6,8 y 12 semanas \\
\hline Mustata, et $\mathrm{al}^{18}$ & $\begin{array}{l}\text { Mustata, et al., ejercicio intradialítico con bicicleta ( } 11 \\
\text { pacientes) }\end{array}$ & $\begin{array}{l}2 \text { veces por } \\
\text { semana }\end{array}$ & 3 meses \\
\hline Konstantinidou, et al ${ }^{19}$ & $\begin{array}{l}\text { Konstantinidou, et al., } 3 \text { programas de rehabilitación: } \\
\text { interdialítico, intradialítico y plan casero no supervisado } \\
\text { con } 1 \text { hora de duración ( } 7 \text { pacientes). }\end{array}$ & $\begin{array}{l}3 \text { veces por } \\
\text { semana }\end{array}$ & No describe \\
\hline Leaf, et $\mathrm{al}^{20}$ & $\begin{array}{l}\text { Leaf, et al., entrenamiento fisico de antebrazo con } \\
\text { contracción isométrica de brazo ( } 5 \text { pacientes). }\end{array}$ & No describe & 6 semanas \\
\hline Headley, et $\mathrm{al}^{21}$ & $\begin{array}{l}\text { Headley, et al., entrenamiento de fuerza para miembros } \\
\text { superiores con circuitos con máquinas ( } 10 \text { pacientes). }\end{array}$ & No describe & 12 semanas \\
\hline Kouidi, et $\mathrm{al}^{22}$ & $\begin{array}{l}\text { Kouidi, et al., (2004), ejercicio aeróbico interdialítico e } \\
\text { intradialítico ( } 48 \text { pacientes). }\end{array}$ & $\begin{array}{l}3 \text { veces por } \\
\text { semana }\end{array}$ & 4 años \\
\hline Kouidi, et $\mathrm{al}^{23}$ & $\begin{array}{l}\text { Kouidi, et al., (1998), entrenamiento aeróbico, natación o } \\
\text { juegos con bola. }\end{array}$ & $\begin{array}{l}3 \text { veces por } \\
\text { semana }\end{array}$ & 6 meses \\
\hline Storer, et $\mathrm{al}^{24}$ & Storer entrenamiento de resistencia ( 20 pacientes). & $\begin{array}{l}3 \text { veces por } \\
\text { semana }\end{array}$ & 9 semanas \\
\hline Painter, et $\mathrm{al}^{26}$ & $\begin{array}{l}\text { Painter, et al., caminata, flexibilidad y fuerza en casa sin } \\
\text { supervisión y ejercicio en cicloergómetro durante la diálisis } \\
\text { ( } 286 \text { paciente). }\end{array}$ & $\begin{array}{l}3-4 \text { veces por } \\
\text { semana }\end{array}$ & no describe \\
\hline Macdonald, et $\mathrm{al}^{27}$ & $\begin{array}{l}\text { Macdonald, et al., entrenamiento intervalado de alta } \\
\text { intensidad y ejercicio de fuerza para hipertrofia muscular ( } 9 \\
\text { pacientes). }\end{array}$ & No describe & 3 meses \\
\hline
\end{tabular}




\section{Intervención}

\section{Tipos y frecuencia del ejercicio}

El tipo de intervención más frecuentemente realizado fue el ejercicio cardiovascular (de predominio aeróbico) y el modo más utilizado fue en el cicloergómetro, seguido por la bicicleta estática o algún sistema de pedales. La caminata solo fue usada cuando el ejercicio se realizó en periodos fuera de la diálisis, siendo no supervisado, como se describe en los estudio de Painter, et al. ${ }^{3}$, en algunos estudios la intervención realizada fue combinada (con ejercicios de predominio aeróbico y también de fuerza), y en otros solo con ejercicios de fuerza (con auto cargas y bandas elásticas principalmente) como en los estudios de Olvera-Soto y Thompson, et al., pero en el estudio de Simo, et al ${ }^{15}$., incluyeron también peso libre (con mancuernas) y otros estudios usaron otras máquinas como en el de Heatly, et $\mathrm{al}^{7-17}$. En tabla 3, se describen más detalladamente.

En aquellos estudios donde se hizo ejercicio durante la hemodiálisis, las intervenciones fueron hechas por un periodo mínimo de 6 a 8 semanas, aunque algunas de estas intervenciones llegaron hasta las 21 y 40 semanas; siendo 12 semanas la duración más usada y solo un estudio (con la mayor duración) fue de 4 años, donde se incluyeron interven-

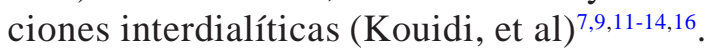

El número de sesiones en la mayoría de los estudios fue de 2 a 3 sesiones por semana, y solo el estudio de Painter menciona de 3 a 4 sesiones semanales ${ }^{9,11-14,16}$. También en la mayoría de estudios, los ejercicios fueron realizados entre la primera y segunda hora de la terapia de reemplazo renal, siendo pocos los estudios que no especifican el momento de la intervención durante el programa de ejercicio $^{7-17}$, tabla 3 .

\section{Intensidad de la intervención y duración de la sesión}

La intensidad del ejercicio durante de las sesiones fue calificada de leve a moderada, medida con la escala subjetiva del esfuerzo "BORG" (en valores de 8 a 17 en escala de 6 a 20), también con porcentajes del consumo de oxígeno máximo (VO2Max) o de la frecuencia cardiaca máxima (FC máx) desde el $40 \%$ hasta el $85 \%{ }^{7,8,13}$. No se reportan intervenciones de alta intensidad en ejercicio de predominio aeróbico o de cargas de alta intensidad en trabajos de fuerza ${ }^{14,17}$ y la duración mínima de las sesiones fue de 15 minutos, pero en algunos casos se reportan duraciones de hasta 60 minutos por sesión $3,7,9$.

\section{Tipo de monitorización de las sesiones}

La forma de monitorización de las sesiones no está reportada en varios de los artículos, y en aquellos que la informan, indican que dicha supervisión durante la sesión fue realizada por personal de salud calificado y en los programas que incluyeron adicionalmente ejercicios domiciliarios, refieren que se hizo contacto a distancia con alguna regularidad para esta supervisión ${ }^{14}$, lo cual además se constituía como una forma para motivar a los participantes de tal forma que se mantuvieran activos en el plan de ejercicios domiciliarios ${ }^{13}$.

\section{Seguridad y eventos adversos}

Son pocos los artículos en los que refieren la presentación de efectos adversos, otros simplemente no los narran. En los estudios de Olvera-Soto, Paglialonga, Simo, et al., se informa: "ningún efecto adverso" en relación a la intervención $n^{9,15,16}$. También en la revisión sistemática de Segura-Ortíz describen que en 4 estudios no se reportó ningún efecto adverso durante el programa de ejercicio ${ }^{14,16}$.

En otros estudios, como en la revisión narrativa de Spinola Najas ${ }^{10,11,13,17}$, informan sobre "Seguridad y eficacia del entrenamiento físico en la enfermedad renal crónica", pero no informan sobre sus efectos adversos, y destacan los cambios hemodinámicos en los pacientes con ERC, que son sometidos a una intervención con ejercicio físico durante la diálisis, siendo similares a los cambios hemodinámicos de personas sanas, recalcando la importancia de la monitorización de la estabilidad hemodinámica ${ }^{12}$.

Las intervenciones como la del estudio de Thompson, et $\mathrm{al}^{7}$, clasifican como eventos adversos 
Tabla 3. Intervenciones y reporte de eventos adversos.

\begin{tabular}{|c|c|c|c|c|}
\hline Estudio & Objetivo & $\begin{array}{c}\text { Tamaño } \\
\text { de muestra }\end{array}$ & Eventos adversos & Resultado \\
\hline Thompson, et al ${ }^{7}$ & $\begin{array}{l}\text { Evaluar la viabilidad de un } \\
\text { estudio principal que evalúe la } \\
\text { eficacia en QoL del ciclismo y } \\
\text { ejercicio de fuerza, cada uno } \\
\text { realizado durante el } \\
\text { tratamiento de hemodiálisis. }\end{array}$ & 25 pacientes & $\begin{array}{l}\text { Complicación } \\
\text { fístula }(n=2) \\
\text { Hipotensión }(n=1) \\
\text { Hipertensión }(n=3) \\
\text { Trauma }(n=1)\end{array}$ & $\begin{array}{l}\text { Ninguno de los eventos } \\
\text { adversos presentados fue } \\
\text { severo y la frecuencia de } \\
\text { presentación fue baja. }\end{array}$ \\
\hline Paglialonga, et $\mathrm{al}^{9}$ & $\begin{array}{l}\text { El ejercicio intradiálisis puede } \\
\text { mejorar Kt } 1 \mathrm{~V} \text {, VO } 2 \text { pico, y } \\
\text { la calidad de vida, el ejercicio } \\
\text { intradiálisis es seguro para los } \\
\text { pacientes con HD. Por lo } \\
\text { tanto, presentamos sugerencia } \\
\text { de actualizar la guía clínica } \\
\text { para informar a los clínicos } \\
\text { sobre los beneficios del } \\
\text { ejercicio intradiálisis en } \\
\text { pacientes con HD. }\end{array}$ & 10 individuos & ninguno & $\begin{array}{l}30 \text { minutos de ejercicios de } \\
\text { ciclismo intradiálisis es } \\
\text { factible para la mayoría de } \\
\text { los pacientes pediátricos en } \\
\text { HD crónica y son bien } \\
\text { aceptados y tolerados. Este } \\
\text { programa de ejercicios } \\
\text { puede conducir a una } \\
\text { mejora de la capacidad de } \\
\text { ejercicio de esta población. }\end{array}$ \\
\hline Oh Par k, et al ${ }^{11}$ & $\begin{array}{l}\text { Investigar la seguridad y } \\
\text { viabilidad del entrenamiento } \\
\text { aeróbico y de fuerza durante la } \\
\text { hemodiálisis para pacientes } \\
\text { con enfermedad renal terminal } \\
\text { y evaluar su impacto en su } \\
\text { condición cardiaca, fuerza } \\
\text { muscular y estado funcional. }\end{array}$ & 22 individuos & No reporta & $\begin{array}{l}\text { Un programa de ejercicio } \\
\text { bien diseñado durante la } \\
\text { hemodiálisis puede llevarse } \\
\text { a cabo con seguridad con la } \\
\text { supervisión adecuada y la } \\
\text { educación del paciente, } \\
\text { mejorar la fuerza muscular, } \\
\text { la función mental y física y } \\
\text { posiblemente aptitud } \\
\text { cardiaca. }\end{array}$ \\
\hline Bohm, et $\mathrm{al}^{13}$ & $\begin{array}{l}\text { Revisar la literatura sobre los } \\
\text { efectos del ejercicio en los } \\
\text { pacientes en hemodiálisis. }\end{array}$ & 541 pacientes & $\begin{array}{l}\text { No reporta eventos } \\
\text { adversos }\end{array}$ & $\begin{array}{l}\text { La evidencia sugiere que } \\
\text { los pacientes en } \\
\text { hemodiálisis deben ser } \\
\text { incluidos en un programa } \\
\text { de ejercicio estandarizado. }\end{array}$ \\
\hline Esteve Simo V., et al ${ }^{15}$ & $\begin{array}{l}\text { Analizar el efecto de un } \\
\text { programa adaptado de } \\
\text { ejercicio físico intradiálisis } \\
\text { sobre } \\
\text { la fuerza muscular, la } \\
\text { capacidad funcional y la } \\
\text { calidad de vida relacionada } \\
\text { con la salud en } \\
\text { nuestros pacientes ancianos } \\
\text { (>80 años) en hemodiálisis. }\end{array}$ & 22 pacientes & Ninguno & $\begin{array}{l}\text { El programa adaptado de } \\
\text { ejercicio físico intradiálisis } \\
\text { mejoró la fuerza muscular, } \\
\text { la capacidad funcional y la } \\
\text { calidad de vida relacionada } \\
\text { con la salud de los } \\
\text { pacientes ancianos en HD. }\end{array}$ \\
\hline Olvera, et al ${ }^{16}$ & $\begin{array}{l}\text { Evaluar el efecto del ejercicio } \\
\text { de fuerza realizado durante las } \\
\text { sesiones de hemodiálisis sobre } \\
\text { los indicadores } \\
\text { antropométricos de la reserva } \\
\text { muscular y la fuerza de la } \\
\text { mano. }\end{array}$ & 61 pacientes & Ninguno & $\begin{array}{l}\text { A favor del programa de } \\
\text { ejercicio. }\end{array}$ \\
\hline $\begin{array}{l}\text { Parsons y King Val } \\
\text { Vlack }{ }^{17}\end{array}$ & $\begin{array}{l}\text { Revisión impacto ejercicio } \\
\text { intradialítico vs. extradialítico }\end{array}$ & 573 pacientes & No reporta & $\begin{array}{l}\text { El ejercicio intradiálisis } \\
\text { mejora más la presión } \\
\text { arterial y la función } \\
\text { vascular en comparación } \\
\text { con el ejercicio } \\
\text { extradiálisis. }\end{array}$ \\
\hline Mustata, et al ${ }^{18}$ & $\begin{array}{l}\text { Ejercicio predialítico con } \\
\text { bicicleta }\end{array}$ & 11 pacientes & No reporta & $\begin{array}{l}\text { Disminución de rigidez } \\
\text { arterial. }\end{array}$ \\
\hline
\end{tabular}


Rev. Colomb. Nefrol. 2019;6(1): 35 - 47, enero - junio de 2019 http://www.revistanefrologia.org http://dx.doi.org/10.22265/acnef.6.1.328

Continuación Tabla 3. Intervenciones y reporte de eventos adversos.

\begin{tabular}{|c|c|c|c|c|}
\hline Estudio & Objetivo & $\begin{array}{l}\text { Tamaño de } \\
\text { muestra }\end{array}$ & Eventos adversos & Resultado \\
\hline Konstantinidou, et al. ${ }^{19}$ & $\begin{array}{l}\text { Tres programas de } \\
\text { rehabilitación: interdialítico, } \\
\text { intradialítico y plan casero no } \\
\text { supervisado con } 1 \text { hora de } \\
\text { duración. }\end{array}$ & 48 pacientes & No reporta & $\begin{array}{l}\text { El ejercicio más favorable } \\
\text { fue el interdialítico, pero los } \\
\text { pacientes prefirieron el } \\
\text { intradialítico, mejoría en } \\
\text { cualidades fisicas en todos } \\
\text { los modelos de } \\
\text { entrenamiento. }\end{array}$ \\
\hline Leaf, et $\mathrm{al}^{20}$. & $\begin{array}{l}\text { Entrenamiento fisico de } \\
\text { miembros superiores con } \\
\text { contracción isométrica de } \\
\text { brazo. }\end{array}$ & 5 pacientes & No reporta & $\begin{array}{l}\text { Aumento del tamaño de la } \\
\text { vena cefálica. }\end{array}$ \\
\hline Headley, et $\mathrm{al}^{21}$ & $\begin{array}{l}\text { Entrenamiento de fuerza para } \\
\text { miembros superiores con } \\
\text { circuitos con máquinas. }\end{array}$ & 10 pacientes & No reporta & $\begin{array}{l}\text { Aumento del V02 pico, } \\
\text { aumento de la distancia en } \\
\text { el test de } 6 \text { minutos, } \\
\text { disminución del tiempo en } \\
\text { el test de sentar/levantar, no } \\
\text { efectos significativos sobre } \\
\text { hipotensión. }\end{array}$ \\
\hline Kouidi, et al ${ }^{22}$ & $\begin{array}{l}\text { Entrenamiento aeróbico, } \\
\text { natación o juegos con bola. }\end{array}$ & 7 pacientes & No reporta & $\begin{array}{l}\text { Aumento en la velocidad de } \\
\text { conducción nerviosa de } \\
\text { fibras tipo II, V02 pico y } \\
\text { fuerza pico en miembros } \\
\text { inferiores. }\end{array}$ \\
\hline Kouidi, et al $^{23}$ & $\begin{array}{l}\text { Ejercicio aeróbico } \\
\text { interdialítico vs. Intradialítico. }\end{array}$ & 48 pacientes & No reporta & $\begin{array}{l}\text { Aumento del V02, mejoría } \\
\text { calidad de vida. }\end{array}$ \\
\hline Storer, et al ${ }^{24}$ & $\begin{array}{l}\text { Entrenamiento en } \\
\text { cicloergómetro. }\end{array}$ & 12 pacientes & No Reporta & $\begin{array}{l}\text { Aumento función } \\
\text { cardiopulmonar, potencia y } \\
\text { fuerza. }\end{array}$ \\
\hline Kong, et $\mathrm{al}^{25}$ & $\begin{array}{l}\text { Sesión única de ejercicio con } \\
\text { cicloergómetro durante } 60 \\
\text { minutos. }\end{array}$ & 11 pacientes & No reporta & $\begin{array}{l}\text { Aumento de la eficiencia } \\
\text { dialítica ( } \mathrm{KtN}) \\
\text { disminución de creatinina, } \\
\text { potasio, efecto rebote de } \\
\text { urea. }\end{array}$ \\
\hline Painter, et $\mathrm{al}^{26}$ & $\begin{array}{l}\text { Ejercicio de resistencia } \\
\text { cardiovascular } 30 \text { minutos, } 4 \\
\text { veces por semana }\end{array}$ & 167 pacientes & No Reporta & $\begin{array}{l}\text { Aumento del V02 pico, } \\
\text { fuerza y mejoría de la } \\
\text { funcionalidad. }\end{array}$ \\
\hline Macdonald, et al ${ }^{27}$ & $\begin{array}{l}\text { Entrenamiento intervalado de } \\
\text { alta intensidad y ejercicio de } \\
\text { fuerza para hipertrofia } \\
\text { muscular. }\end{array}$ & 9 pacientes & No reporta & $\begin{array}{l}\text { Aumento de la capacidad } \\
\text { fisica sin reversión de la } \\
\text { atrofia muscular. }\end{array}$ \\
\hline Cheema et $\mathrm{al}^{28}$ & $\begin{array}{l}\text { Impacto de entrenamiento de } \\
\text { fuerza en la calidad y volumen } \\
\text { muscular. }\end{array}$ & 49 pacientes & $\begin{array}{l}\text { Cefalea, hipotensión, } \\
\text { calambres, } \\
\text { dificultades con la } \\
\text { fistula. } \\
\text { Desgarro manguito } \\
\text { rotador. }\end{array}$ & $\begin{array}{l}\text { No se encontró diferencia } \\
\text { estadisticamente } \\
\text { significativa en cuanto a } \\
\text { eventos adversos entre el } \\
\text { grupo de intervención y el } \\
\text { grupo control. }\end{array}$ \\
\hline DePaul, et al ${ }^{29}$ & $\begin{array}{l}\text { Efecto de un programa de } \\
\text { ejercicio de resistencia más } \\
\text { fortalecimiento progresivo en } \\
\text { pacientes en hemodiálisis y } \\
\text { manejo con eritropoyetina. }\end{array}$ & 38 pacientes & $\begin{array}{l}\text { Retiro por fatiga, } \\
\text { hipotensión, abrasión } \\
\text { con pedal. Dolor en } \\
\text { extremidades por } \\
\text { fortalecimiento. }\end{array}$ & $\begin{array}{l}\text { No diferencia estadística } \\
\text { entre los grupos en los } \\
\text { parámetros de laboratorio } \\
\text { medidos, todos los eventos } \\
\text { adversos fueron leves. }\end{array}$ \\
\hline Koufaki, et al ${ }^{30}$ & $\begin{array}{l}\text { Efecto del ejercicio en la } \\
\text { capacidad aeróbica y funcional } \\
\text { en pacientes con enfermedad } \\
\text { renal crónica en estadío } \\
\text { terminal. }\end{array}$ & 33 pacientes & $\begin{array}{l}\text { Ruptura ligamento de } \\
\text { la rodilla (no } \\
\text { relacionado con el } \\
\text { protocolo) }\end{array}$ & $\begin{array}{l}\text { No se evidenciaron } \\
\text { complicaciones } \\
\text { relacionadas con el } \\
\text { protocolo de ejercicio. }\end{array}$ \\
\hline
\end{tabular}


Tabla 4. Características de las poblaciones intervenidas.

\begin{tabular}{|c|c|c|c|c|c|}
\hline Articulo & $\begin{array}{c}\text { Hb }(\mathrm{g} / \mathrm{dL}) \mathrm{o} \\
\text { Heto }(\%)\end{array}$ & Albumina & $\begin{array}{c}\text { FEVI } \\
\%\end{array}$ & Otros & $\begin{array}{c}\text { Características de la población } \\
\text { intervenida }\end{array}$ \\
\hline Leaf, et $\mathrm{al}^{20}$ & $33,7 \pm 5,3 \%$ & N.R & N.R & $\begin{array}{l}\text { Creatinina } 3,8 \pm 1,5 \\
\mathrm{TFG}=33,7 \pm 5,3 \mathrm{~mL} / \mathrm{min}\end{array}$ & $\begin{array}{l}\mathrm{n}=5, \text { edad media } 57 \pm 9, \text { peso } \\
\text { medio } 80,0 \pm 5,1\end{array}$ \\
\hline Headley, et $\mathrm{al}^{21}$ & N.R & N.R & N.R & $\begin{array}{l}\text { TFG }=30-59 \mathrm{~mL} / \mathrm{min} \text { por } \\
1,73 \mathrm{~m} 2\end{array}$ & $\begin{array}{l}\mathrm{n}=46, \text { edad media } 58 \pm 8, \text { peso } \\
\text { medio } 101,7 \pm 24,9\end{array}$ \\
\hline Kouidi, et $\mathrm{al}^{22}$ & $30,9 \pm 4,2 \%$ & N.R & N.R & Creatinina $13,2 \pm 4,0$ & $\begin{array}{l}\mathrm{n}=7, \text { edad media } 44,1 \pm 17.2, \text { peso } \\
\text { medio } 67,0 \pm 15.9\end{array}$ \\
\hline Storer, et $\mathrm{al}^{24}$ & $11,4 \pm 1,4 \mathrm{~g} / \mathrm{dL}$ & N.R & N.R & Creatinina $12,5 \pm 3,5$ & $\begin{array}{l}\mathrm{n}=12, \text { edad media } 44 \pm 9, \text { peso } \\
\text { medio } 76 \pm 12\end{array}$ \\
\hline Kong, et $\mathrm{al}^{25}$ & 9,5 a $13,9 \mathrm{~g} / \mathrm{dL}$ & N.R & N.R & Creatinina 0,57 & $\mathrm{n}=11$, edad 32 a 78 \\
\hline Painter, et $\mathrm{al}^{26}$ & N.R & N.R & N.R & N.R & $\mathrm{n}=56$, edad $39,7 \pm 12,6$ \\
\hline Macdonald, et $\mathrm{al}^{27}$ & $11,7 \pm 0,4 \mathrm{~g} / \mathrm{dL}$ & $41,3 \pm 1,8 \mathrm{~g} / \mathrm{L}$ & N.R & N.R & $\begin{array}{l}\mathrm{n}=9, \text { edad media } 48,4 \pm 5,3, \mathrm{IMC}= \\
24,8 \pm 1,5\end{array}$ \\
\hline Cheema, et al., $2007^{28}$ & N.R & $34.5 \pm 3.1 \mathrm{~g} / \mathrm{L}$ & N.R & $\begin{array}{l}\text { Creatinina } 940,9 \pm 185,9 \text { ų } \\
\mathrm{mol} / \mathrm{L}\end{array}$ & $\begin{array}{l}\mathrm{n}=49, \text { edad media } 62,6 \pm 14,2, \\
\text { peso medio } 75.7 \pm 18,3\end{array}$ \\
\hline DePaul, et al., $2002^{29}$ & $\begin{array}{c}11,6 \pm 1,2 \mathrm{~g} / \mathrm{dL} \\
35 \pm 4 \%\end{array}$ & N.R & N.R & $\begin{array}{l}\text { Creatinina } \\
814,9 \pm 176,5 u \underline{\mathrm{mol}} / \mathrm{L}\end{array}$ & $\mathrm{n}=20$, edad media $55 \pm 16$ \\
\hline Koufaki, et $\mathrm{al}^{30}$ & $12,1 \pm 1,4 \mathrm{~g} / \mathrm{dL}$ & $39 \pm 5,5 \mathrm{~g} / \mathrm{L}$ & N.R & N.R & $\begin{array}{l}\mathrm{n}=18, \text { edad media } 57,3 \pm 14,3 \\
\text { peso medio } 76,3 \pm 13,6\end{array}$ \\
\hline
\end{tabular}

$\mathrm{Hb}=$ hemoglobina, Hcto $=$ hematocrito, N.R = No registra, $\mathrm{TFG}=$ Tasa de filtración glomerular, $\mathrm{n}=$ número de participantes en intervención, IMC= Indice de masa corporal.

serios a cualquier evento que amenace la vida, tales como muerte súbita, evento cardiaco, otros que requieran hospitalización o que produzcan alguna discapacidad, y como eventos adversos leves, la lesión músculo esquelética, hipoglicemia, hipotensión, urgencia hipertensiva, alteración del estado de conciencia o que necesiten alguna intervención adicional por parte del personal de la unidad renal diferente a la ultrafiltración. Ellos indican los siguientes resultados de eventos adversos por grupos: en el grupo de ejercicio combinado (aeróbico y de fuerza), y en el grupo de ejercicio aeróbico: dos pacientes presentaron efectos adversos. En el grupo de ejercicio de fuerza: un paciente presentó evento adverso y por último en el grupo de estiramientos: no presentaron eventos adversos. Ninguno de los eventos adversos presentados fue considerado severo, lo que indica que la presentación de estos es muy baja (detalles de los eventos adversos en la tabla 3$)^{7}$.

En la revisión sistemática y meta-análisis de Sheng, se mencionan 3 estudios $\left(\mathrm{Cheema}^{28}\right.$, De Paul $^{29}$ y Koufaki ${ }^{30}$, y en ellos se tienen casos y controles, documentándose efectos adversos con la intervención de ejercicio durante la diálisis, y comparados con el control, se muestra que las complicaciones musculo-esqueléticas (reportadas en los 3 estudios), y las complicaciones cardiovasculares (todas relacionadas con hipotensión) son leves; en este último estudio (de Cheema ${ }^{28}$ ) también se presentó un efecto adverso cardiovascular en el grupo control. (Tablas 3 y 4 )

\section{Conclusiones}

Al revisar la literatura encontramos que la población en la cual se realizaron los estudios eran en su mayoría personas adultas (mayores de 18 años y poblaciones mayores de 80 años), de ambos géneros, cuya causa de la enfermedad renal crónica en su mayoría era por HTA, DM, la enfermedad poliquística renal y la enfermedad glomerular, y cuyos criterios de exclusión para participar en los estudios eran definidos en su gran mayoría relacionados por tener o haber tenido recientemente alguna enfermedad aguda (en su mayoría de origen coronario) o que presenten contraindicaciones para realizar una prueba de esfuerzo según las normas de la Sociedad Americana del Corazón (AHA), donde el riesgo a presentar un evento cardiaco agudo esta aumentado. Los cri- 
terios de inclusión en general se refieren a poblaciones controladas en un programa de HD (mayor a tres meses consecutivos y con sesiones de 3 o más veces por semana) sin otras consideraciones o variables bioquímicas que contraindican la realización de una actividad física dirigida y controlada, y cuya intensidad sea moderada. Se encuentra que la seguridad de la intervención con ejercicio durante la hemodiálisis es segura, y en aquellos estudios que reportan eventos adversos, estos son leves en su mayoría, y en aquellos que se reportan como graves se presentaron tanto en el grupo de intervención con ejercicio físico como en los controles en una misma proporción sin representar una diferencia estadísticamente significativa, por lo cual podemos concluir que con la evidencia disponible se puede recomendar una intervención con ejercicio físico intrahemodiálisis como una modalidad terapéutica segura.

\section{Discusión}

Encontramos que en muchos estudios no está reportada la incidencia de eventos adversos asociados a la intervención con ejercicio durante la hemodiálisis, ya sea porque no se presentaron o por que no se hizo ninguna referencia a las complicaciones o efectos adversos relacionados con la intervención, ya que simplemente aquellos que se presentaron son de común ocurrencia durante la diálisis. Para estos programas de ejercicio intradiálisis, en general, se incluye todo tipo de población (excluyendo según algunos artículos revisados a las poblaciones con un alto riesgo de presentar complicaciones cardiacas relacionadas con ejercicios de alta intensidad, como sería durante una prueba de esfuerzo cardiovascular, pero no lo contraindican en ejercicios de moderada o baja intensidad), con un rango etario donde predominan las personas mayores de edad, siendo pocas las intervenciones en niños y adolescentes. Con respecto a las sesiones de ejercicio, estas son de baja a moderada intensidad, encontrándose que no hay estudios con intensidades o cargas altas, tanto de ejercicio cardiovascular como de fuerza. Los estudios que reportaron efectos adversos, fueron clasificados como no severos y de baja frecuencia en su presentación, lo que permite intuir que es una intervención segura para esta población. En este estudio se in- cluyeron revisiones narrativas, pero también revisiones sistemáticas y meta-análisis que proporcionan datos relevantes para nuestro objetivo, aunque no se dispone de una gran cantidad de estudios clínicos que enuncien efectos adversos que se puedan relacionar con este tipo de programas, por este motivo, se hace muy importante darle relevancia a la seguridad de estas actividades durante un programa de hemodiálisis, para que en futuros estudios se puedan brindar recomendaciones con un alto poder de evidencia y de una estadística que demuestre la seguridad del ejercicio físico intrahemodiálisis.

\section{Contribución de los autores}

Angélica puentes y Andrés Hernández realizaron la búsqueda sistemática de artículos, los tres autores realizaron búsqueda manual de artículos.

Los tres autores revisaron los artículos seleccionados. Oscar Sarmiento construyó las tablas.

Todos los autores revisaron el escrito final y dieron su aprobación.

\section{Agradecimientos}

Agradecemos a la División de Investigaciones por su asesoría.

\section{Conflicto de interés}

Los autores del presente artículo declaran no tener ningún conflicto de interés

\section{Declaración de financiación del proyecto}

Esta revisión no requirió financiación interna o externa.

\section{Responsabilidades éticas}

\section{Protección de personas y animales}

Los autores declaran que para esta investigación no se han realizado experimentos en seres humanos ni en animales. 


\section{Confidencialidad de los datos}

Los autores declaran que han seguido los protocolos de su centro de trabajo sobre la publicación de datos de pacientes.
Derecho a la privacidad y consentimiento formado

Los autores declaran que en este artículo no aparecen datos de pacientes. 


\section{Rev. Colomb. Nefrol. 2019;6(1): 35 - 47, enero - junio de 2019 http://www.revistanefrologia.org}

http://dx.doi.org/10.22265/acnef.6.1.328

\section{Referencias}

1. Fondo Colombiano de Enfermedades de Alto Costo. Situación de la enfermedad renal crónica, hipertensión arterial y diabetes mellitus en Colombia. Colombia: Fondo Colombiano de Enfermedades de Alto Costo; 2017. p. 79.

2. Lopera-Medina MM. La enfermedad renal crónica en Colombia: necesidades en salud y respuesta del Sistema General de Seguridad Social en Salud. Rev Gerenc Polít Salud. 2016;15(30):212-33. https://doi.org/10.11144/Javeriana.rgyps15-30.ercc

3. Painter P. Implementing exercise: what do we know? Where do we go? Adv Chronic Kidney Dis. 2009;16(6):536-44. https://doi.org/10.1053/j.ackd.2009.07.010

4. Hernández A, Mongui Y, Rojas Y. Descripción de la composición corporal, fuerza muscular y actividad física en pacientescon insuficiencia renal crónica en hemodiálisis en una unidad renal en Bogotá, Colombia. Rev Andal Med Deporte. 2018;11(2):56-52. https://doi.org/10.1016/j.ramd.2016.09.005

5. Jhamb M, McNulty ML, Ingalsbe G, Childers JW, Schell J, Conroy MB, et al. Knowledge, barriers and facilitators of exercise in dialysis patients: a qualitative study of patients, staff and nephrologists. BMC Nephrol. 2016;17(1):192. https://doi.org/10.1186/s12882-0160399-z

6. Johansen KL. Exercise in the end-stage renal disease population. J Am Soc Nephrol. 2007;18(6):1845-54. https://doi.org/10.1681/ ASN.2007010009

7. Thompson S, Klarenbach S, Molzahn A, Lloyd A, Gabrys I, Haykowsky M, et al. Randomised factorial mixed method pilot study of aerobic and resistance exercise in haemodialysis patients: DIALY-SIZE!. BMJ Open. 2016;6(9):e012085. https://doi.org/10.1136/ bmjopen-2016-012085

8. Sheng K, Zhang P, Chen L, Cheng J, Wu C, Chen J. Intradialytic exercise in hemodialysis patients: a systematic review and metaanalysis. Am J Nephrol. 2014;40(5):478-90. https://doi.org/10.1159/000368722

9. Paglialonga F, Lopopolo A, Scarfia RV, Consolo S, Galli MA, Salera S, et al. Intradialytic cycling in children and young adults on chronic hemodialysis. Pediatr Nephrol. 2014;29(3):431-8. https://doi.org/10.1007/s00467-013-2675-5

10. Smart N, Steele M. Exercise training in haemodialysis patients: a systematic review and meta-analysis. Nephrology (Carlton). 2011;16(7):626-32. https://doi.org/10.1111/j.1440-1797.2011.01471.x

11. Oh-Park M, Fast A, Gopal S, Lynn R, Frei G, Drenth R, et al. Exercise for the dialyzed: aerobic and strength training during hemodialysis. Am J Phys Med Rehabil. 2002;81(11):814-21. https://doi.org/10.1097/01.PHM.0000030623.81541.DA

12. Spínola Najas C, Mungo Pissulin FD, Lopes Pacagnelli F, Navarro Betônico G, Costa Almeida I, Alberto Neder J. Segurança e Eficácia do Treinamento Físico na Insuficiência Renal Crônica Rev Bras Med Esporte. 2009;15(5). https://doi.org/10.1590/S151786922009000600013

13. Böhm J, Monteiro MB, Thomé FS. [Effects of aerobic exercise during haemodialysis in patients with chronic renal disease: a literature review]. J Bras Nefrol. 2012;34(2):189-94. https://doi.org/10.1590/S1517-86922009000600013

14. Segura-Ortí E. Ejercicio en pacientes en hemodiálisis: revisión sistemática de la literatura. Revista Nefrología. 2010;30(2):46. https:/ /doi.org/10.3265/Nefrologia.pre2010.Jan.10229

15. Esteve Simo V, Junqué Jiménez A, Moreno Guzmán F, Carneiro Oliveira J, Fulquet Nicolas M, Pou Potau M, et al. Benefits of a low intensity exercise programme during haemodialysis sessions in elderly patients. Nefrologia. 2015;35(4):385-94. https://doi.org/10.1016/ j.nefro.2015.03.006

16. Olvera-Soto MG, Valdez-Ortiz R, López Alvarenga JC, Espinosa-Cuevas MeL. Effect of Resistance Exercises on the Indicators of Muscle Reserves and Handgrip Strength in Adult Patients on Hemodialysis. J Ren Nutr. 2016;26(1):53-60. https://doi.org/10.1053/ j.jrn.2015.06.006

17. Parsons TL, King-Vanvlack CE. Exercise and end-stage kidney disease: functional exercise capacity and cardiovascular outcomes. Adv Chronic Kidney Dis. 2009;16(6):459-81. https://doi.org/10.1053/j.ackd.2009.08.009

18. Mustata S. Groeneveld S. Davidson W. Ford G. Kiland K, et al. Effects of exercise training on physical impairment, arterial stiffness and health-related quality of life in patients with chronic kidney disease: a pilot study. Int Urol Nephrol. (2011) 43:1133-1141. https://doi.org/10.1007/s11255-010-9823-7

46 Seguridad del ejercicio intrahemodiálisis 
19. Konstantinidou E. Koukouvou G. Kouidi E. Deligiannis A. Turkantonis A. Exercise training in patients with end stage renal desease on hemodialysis: comprarison of three rehabilitation programs. J Rehabil Med. 2002;34:(1):40-5.

20. Leaf DA. MacRae HS. Grant E. Kraut J. Isometric Exercise increases the size of forearm veins in patients with chronic renal failure. Am J med Sci 2003; 325(3):115-9.

21. Headley S. Germain M. Wood R. Joubert J. Milch C., et al. Blood pressure response to acute and chronic exercise in chronic kidney disease. Nephrology (Carlton). 2017;22(1):72-78. https://doi.org/10.1111/nep.12730

22. Kouidi E. Grekas D. Deligiannis A. Tourkantonis A. Outcomes of long-term exercise training on muscle atrophy in haemodialysis patients. Nephrol Dial Transplant. 1998;13(3):685-99.

23. Kouidi E. Albani M. Natsis K. Megalopoulos A. Gigis P., et al. The effects of exercise training, in dialysis patients: comparison of two training programs. Clin Nephrol. 2004;61 Suppl1:S31-8.

24. Storer TW. Casaburi R. Sawelson S. Kopple JD. Endurance exercise training during haemodialysis improves strength, power, fatigability and physical performance in maintenance haemodialysis patients. Nephrol Dial Transplant. 2005;20(7):1429-37. https://doi.org/10.1093/ndt/gfh784

25. Chiew H, James T, Roger N, Ken F. The effect of exercise during a haemodialysis on solute removal. Nephrol Dial Transplant. 1999;14:2927-2931. https://doi.org/10.1093/ndt/14.12.2927

26. Painter PL, Hector L, Ray K, Lynes L, Dibble S., et al. A randomized trial of exercise training after renal transplantation. Transplantation. 2002,15;74(1):42-8.

27. Macdonald JH, Marcora SM, Jibani M, Phanish MK, Holly J, et al. Intradialytic exercise as anabolic therapy in haemodialysis patients — a pilot study. Clin Physiol Funct Imaging. 2005;25(2):113-8. https://doi.org/10.1111/j.1475-097X.2004.00600.X

28. Cheema B, Abas H, Smith B, O’Sullivan A, Chan M., et al. Progressive exercise for anabolism in kidney disease (PEAK): a randomized, controlled trial of resistance training during hemodialysis. J Am Soc Nephrol. 2007;18(5):1594-601. https://doi.org/10.1681/ ASN.2006121329

29. DePaul V, Moreland J, Eager T, Clase CM. The effectiveness of aerobic and muscle strength training in patients receiving hemodialysis and EPO: a randomized controlled trial. Am J Kidney Dis. 2002;40(6):1219-29. https://doi.org/10.1053/ajkd.2002.36887

30. Koufaki P, Mercer TH, Naish PF. Effects of exercise training on aerobic and functional capacity of end-stage renal disease patients. Clin Physiol Funct Imaging. 2002;22(2):115-24. 